\title{
A FREE BOUNDARY VALUE PROBLEM RELATED TO THE COMBUSTION OF A SOLID: FLUX BOUNDARY CONDITIONS
}

\author{
BY \\ JOHN R. CANNON (University of Central Florida, Orlando, Florida) \\ AND \\ ALEC L. MATHESON (Lamar University, Beaumont, Texas)
}

Abstract. We demonstrate the existence, uniqueness, and continuous dependence upon the data for the solution $(u, v, s)$ of the free boundary value problem:

$$
\begin{gathered}
u_{t}=\alpha u_{x x}, \quad v_{t}=\beta v_{x x}, \quad 0<x<s(t), \quad 0<t \leq T, \\
u(x, 0)=\phi(x), \quad v(x, 0)=\psi(x), \quad 0 \leq x \leq s(0)=b . \\
-\alpha u_{x}(0, t)=f(t), \quad-\beta v_{x}(0, t)=g(t), \quad 0<t \leq T, \\
\alpha u_{x}(s(t), t)=-(\gamma+u(s(t), t)) \dot{s}(t), \quad \beta v_{x}(s(t), t)=(\mu-v(s(t), t)) \dot{s}(t), \quad 0<t \leq T, \\
\dot{s}(t)=\nu(v(s(t), t)) \exp (-\delta / v(s(t), t)) F(u(s(t), t)), \quad 0<t \leq T,
\end{gathered}
$$

where $\alpha, \beta, \gamma, \delta$, and $\mu$ are positive constants related to the physical constants.

1. Introduction. We shall consider an idealized model for the combustion/oxidation of a half-space full of solid fuel by a gaseous oxidizer contained in an infinite strip bounded by the fuel and a plane. We shall neglect heat conduction in the solid, evaporation of the solid, and compressibility and convection in the gas. Hence, we shall consider the diffusion of the oxidizer, heat conduction in the oxidizer, and the chemical reaction between the oxidizer and fuel at the surface of the fuel. In particular, corrosion would be a close application of the model below. We remark that this work represents a continuation of the study of this problem which began with the paper of Cannon, Cavendish, and Fasano $[\mathrm{CCF}]$ discussing the problem of a half-space of fuel. Fasano $[\mathrm{F}]$ developed some special solutions for a half-space of oxidizer vs. a half-space of fuel problem. Cannon and Lin [CL] discussed the first boundary-value problem for an infinite strip of oxidizer vs. a half-space of fuel with the specification of oxidizer concentration and temperature on the

Received December 1, 1994.

1991 Mathematics Subject Classification. Primary 35K, 35R, 35R35, 80A22, 80A25.

This work was supported in part by the National Science Foundation Grant DMS-9401847. 
fixed boundary of the oxidizer region. Recently, Liang and Cheng [LC] have discussed the case of two free boundaries which appears to consider the case of oxidation of two half-spaces of fuel.

Let the positive $x$-axis be directed normally to the gas-solid interface and into the solid fuel. At time $t=0$, we assume that the gas-solid interface is at $x=b>0$. Assuming that the oxidizer concentration and the temperature depend only on the real variables $x$ and $t$, we see that the gas-solid interface moves as $x=s(t), 0<t \leq T$, with $s(0)=b$. We shall let the plane $x=0$ denote the fixed boundary of the oxidizer region. Let $u=u(x, t)$ denote the concentration of the oxidizer while $v=v(x, t)$ denotes its temperature. Then we are led to consider the free boundary value problem of determining $u=u(x, t)$, $v=v(x, t)$, and $s=s(t)$ which satisfy

$$
\begin{array}{ll}
u_{t}=\alpha u_{x x}, & 0<x<s(t), 0<t \leq T, \\
v_{t}=\beta v_{x x}, & 0<x<s(t), 0<t \leq T, \\
u(x, 0)=\phi(x), & 0 \leq x \leq s(0)=b, \\
v(x, 0)=\psi(x), & 0 \leq x \leq s(0)=b, \\
-\alpha u_{x}(0, t)=f(t), & 0<t \leq T, \\
-\beta v_{x}(0, t)=g(t), & 0<t \leq T, \\
\alpha u_{x}(s(t), t)=-(\gamma+u(s(t), t)) \dot{s}(t), & 0<t \leq T, \\
\beta v_{x}(s(t), t)=(\mu-v(s(t), t)) \dot{s}(t), & 0<t \leq T,
\end{array}
$$

and

$$
\left\{\begin{array}{l}
\dot{s}(t)=\nu\left(v(s(t), t) \exp \left(-\frac{\delta}{v(s(t), t)}\right) F(u(s(t), t)), \quad 0<t \leq T\right. \\
s(0)=b
\end{array}\right.
$$

where $\alpha, \beta, \delta, \gamma$, and $\mu$ are positive constants related to the physical constants, $\phi, \psi, f$, and $g$ are known nonnegative functions, $F$ is a strictly increasing function with $F(0)=0$, $\nu$ is a positive function, and $\dot{s}(t)$ denotes the derivative of $s(t)$. Also, we assume that all functions are real-valued and a subscripted independent variable denotes a partial derivative; e.g., $u_{t}=\frac{\partial u}{\partial t}, u_{x}=\frac{\partial u}{\partial x}, u_{x x}=\frac{\partial^{2} u}{\partial x^{2}}$, etc.

The boundary conditions $(1.1 \mathrm{~g})$ and $(1.1 \mathrm{~h})$ need some explanation. The term $\gamma \dot{s}(t)$ denotes the number of moles per unit time of oxidizer that are transported by diffusion to the boundary for the reaction. The term $u(s(t), t) \dot{s}(t)$ is the mass flux induced by the motion of the boundary in order to preserve conservation of mass. The term $\mu \dot{s}(t)$ denotes the heat energy released per unit time by the reaction while the term $v(s(t), t) \dot{s}(t)$ is the energy flux induced by the motion of the boundary in order to preserve conservation of energy.

Definition. A solution to (1.1)-(1.2) is a triple $(u, v, s)$ that satisfies the following:

(i) $u_{t}, u_{x x}, v_{t}$, and $v_{x x}$ are continuous in $0<x<s(t), 0<t \leq T$;

(ii) $u_{x}$ and $v_{x}$ are continuous in $0 \leq x \leq s(t), 0<t \leq T$;

(iii) $u$ and $v$ are continuous and bounded in $0 \leq x \leq s(t), 0 \leq t \leq T$;

(iv) $s$ is continuous on $0 \leq t \leq T$ and continuously differentiable on $0<t \leq T$;

(v) the triple $(u, v, s)$ satisfies (1.1)-(1.2) with the given data. 
It is convenient to state some assumptions on the data $\phi, \psi, f, g, \nu$, and $F$ that we will use throughout this article.

Assumption A. We shall assume that the functions $\phi$ and $\psi$ are continuous on $0 \leq x \leq b$ and that there exist positive constants $\phi_{*}, \phi^{*}, \psi_{*}$, and $\psi^{*}$ such that

$$
0<\phi_{*} \leq \phi(x) \leq \phi^{*}, \quad 0<\psi_{*} \leq \psi(x) \leq \psi^{*} .
$$

Assumption B. We shall assume that $f$ and $g$ are continuous and nonnegative on $0 \leq$ $t \leq T$.

We let

$$
\begin{cases}f^{*}=\max _{0 \leq t \leq T} f(t), & f_{*}=\min _{0 \leq t \leq T} f(t), \\ g^{*}=\max _{0 \leq t \leq T} g(t), & g_{*}=\min _{0 \leq t \leq T} g(t) .\end{cases}
$$

Assumption C. We shall assume that the functions $F$ and $\nu$ are continuously differentiable on $-\infty<u<\infty, 0 \leq v<\infty$, respectively, and that $F(0)=0$, that

$$
F^{\prime}(u)>0
$$

and that $\nu(0) \geq 0$ and

$$
\nu^{\prime}(v)>0 .
$$

If $f$ and $g$ are continuous in $t$ for $0 \leq t \leq T$ and $\phi$ and $\psi$ are continuously differentiable on $0 \leq x \leq b$, then we obtain a priori bounds on $u_{x}$ and $v_{x}$ that are uniform in $t[\mathrm{C}]$.

Assumption D. We shall assume that the functions $f$ and $g$ are continuous on $0 \leq t \leq$ $T$, and that the functions $\phi$ and $\psi$ are continuously differentiable on $0 \leq x \leq b$.

The main purpose of this article is to demonstrate the following result.

Theorem. Under assumptions $\mathrm{A}, \mathrm{B}$, and $\mathrm{C}$, there exists a solution $(u, v, s)$ to problem (1.1)-(1.2). Under the addition of assumption $\mathrm{D}$, the solution is unique and depends continuously upon the data.

2. A priori estimates. We shall derive some a priori estimates of a solution $(u, v, s)$ to (1.1)-(1.2). Using the parabolic version of Hopf's lemma [C, FR] and the argument in $[\mathrm{CCF}]$ in $[\mathrm{CL}]$ we can state the following result.

Lemma 2.1. For $0 \leq x \leq s(t), 0 \leq t \leq T, u(x, t)>0, v(x, t)>0$, and $\dot{s}(t)>0$.

Proof. Omitted.

We turn now to an a priori upper estimate of $u$. Set $u=w+z$, where

$$
\begin{array}{ll}
w_{t}=\alpha w_{x x}, & 0<x<s(t), 0<t \leq T, \\
w(x, 0)=\phi(x), & 0 \leq x \leq b=s(0), \\
-\alpha w_{x}(0, t)=0, & 0<t \leq T, \\
\alpha w_{x}(s(t), t)=-(\gamma+u(s(t), t)) \dot{s}(t), & 0<t \leq T,
\end{array}
$$


and

$$
\begin{array}{ll}
z_{t}=\alpha z_{x x}, & 0<x<s(t), 0<t \leq T, \\
z(x, 0)=0, & 0 \leq x \leq b=s(0), \\
-\alpha z_{x}(0, t)=f(t), & 0<t \leq T, \\
\alpha z_{x}(s(t), t)=0, & 0<t \leq T .
\end{array}
$$

We note that the assumption of the existence of $(u, v, s)$ implies that $\dot{s}(t)$ is continuous and bounded. Lemma 2.1 implies that $s(t)$ is strictly increasing. Hence, by standard parabolic theory [C], the existence and uniqueness of $w$ and $z$ follow. Elementary maximum principle arguments show that the function $\zeta=u-(w+z) \equiv 0$. Hence, to estimate $u$, it suffices to estimate $w$ and $z$.

We begin with $z$. By the standard theory for the heat equation, it follows that $z_{x}$ is bounded and continuous in $0 \leq x \leq s(t), 0<t \leq T$, and $0<x \leq b, t=0$. By the strong maximum principle $z_{x}<0$ in $0 \leq x<s(t), 0<t \leq T$. Consequently, $z$ takes its maximum value on $x=0,0<t$. Also, $z \geq 0$. Otherwise, $z$ would have to assume a negative minimum on $x=0$ or $x=s(t)$. By the parabolic version of Hopf's lemma, $z_{x}(0, t)>0$ or $z_{x}(s(t), t)<0$. Either of these conditions contradicts $(2.2 \mathrm{c})$ or $(2.2 \mathrm{~d})$. In order to bound $z$, we consider the comparison function $\eta$ which satisfies

$$
\begin{array}{ll}
\eta_{t}=\alpha \eta_{x x}, & 0<x<b, 0<t \leq T, \\
\eta(x, 0)=0, & 0 \leq x \leq b, \\
-\alpha \eta_{x}(0, t)=f(t), & 0<t \leq T, \\
\eta_{x}(b, t)=0, & 0<t \leq T .
\end{array}
$$

Set $\zeta=\eta-z$. Then $\zeta$ satisfies

$$
\begin{array}{ll}
\zeta_{t}=\alpha \zeta_{x x}, & 0<x<b, 0<t \leq T, \\
\zeta(x, 0)=0, & 0 \leq x \leq b, \\
-\alpha \zeta_{x}(0, t)=0, & 0<t \leq T, \\
\zeta_{x}(b, t)=-z_{x}(b, t)>0, & 0<t \leq T,
\end{array}
$$

Suppose $\zeta<0$. Then, from the maximum principle $\zeta$ would assume a negative minimum on $x=0$ or $x=b$. Again we would have either $\zeta_{x}(0, t)>0$ or $\zeta_{x}(b, t)<0$ which contradict $(2.4 \mathrm{c})$ or $(2.4 \mathrm{~d})$ respectively. Thus, $\zeta \geq 0$ from whence follows $\eta(0, t) \geq z(0, t)$. Hence, it suffices to estimate $\eta(0, t)$ in order to bound $z$. For this purpose, we consider the comparison function

$$
y(x, t)=B t+C(x-b)^{2} .
$$

This time set $\zeta=y-\eta$. Then

$$
\begin{array}{ll}
\zeta_{t}-\alpha \zeta_{x x}=B-2 \alpha C, & 0<x<b, 0<t \leq T, \\
\zeta(x, 0)=C(x-b)^{2} \geq 0, & 0 \leq x \leq b, \\
-\alpha \zeta_{x}(0, t)=2 \alpha b C-f(t), & 0<t \leq T \\
\zeta_{x}(b, t)=0, & 0<t \leq T .
\end{array}
$$


By selecting

$$
C=(2 \alpha b)^{-1} f^{*}
$$

and

$$
B=b^{-1} f^{*}+\varepsilon, \quad \varepsilon>0,
$$

it follows that $\zeta \geq 0$. Indeed, suppose that $\zeta<0$. From the parabolic version of Hopf's lemma, (2.6b), (2.6c), and (2.6d) imply that $\zeta$ would have to assume its negative minimum at $\left(x_{0}, t_{0}\right)$, where $0<x_{0}<b$ and $0<t_{0} \leq T$. Thus the negative minimum at $\left(x_{0}, t_{0}\right)$ together with (2.6a) imply

$$
0 \geq \zeta_{t}-\alpha \zeta_{x x}=\varepsilon>0
$$

which is a contradiction. Hence, $\zeta \geq 0$ for all $\varepsilon>0$ and also for $\varepsilon=0$. Thus

$$
0 \leq z(x, t) \leq \eta(0, t) \leq\left(b^{-1} T+(2 \alpha b)^{-1}\right) f^{*}
$$

for $0 \leq x \leq s(t)$ and $0 \leq t \leq T$.

Turning now to $w(x, t)$, we see that $w_{x}(0, t)=0$ and $w_{x}(s(t), t)<0$. Hence, the parabolic version of Hopf's lemma implies that $w(x, t)$ cannot assume a maximum value on $x=0$ or $x=s(t)$. Thus, the maximum principle implies that $w$ assumes its maximum value on $t=0$. Hence

$$
w(x, t) \leq \phi^{*} .
$$

Combining $u=w+z$ with (2.10) and (2.11) we obtain the following result.

LEMMA 2.2. For $0 \leq x \leq s(t), 0 \leq t \leq T$,

$$
0<u(x, t) \leq \phi^{*}+\left(b^{-1} T+(2 \alpha b)^{-1}\right) f^{*} .
$$

Proof. See the analysis preceding the statement of the lemma.

As a corollary of the argument of Lemma 2.1, it follows from $v_{x}(0, t)=-\beta^{-1} g(t)$ that the minimum of $v$ in $0 \leq x \leq s(t), 0 \leq t \leq T$ cannot occur on $x=0$. By the maximum principle it must occur on $t=0$ or $x=s(t)$. If it occurs on $x=s(t)$ at $\left(s\left(t_{0}\right), t_{0}\right)$, then the parabolic version of Hopf's lemma implies that

$$
0>\beta v_{x}\left(s\left(t_{0}\right), t_{0}\right)=\left(\mu-v\left(s\left(t_{0}\right), t_{0}\right)\right) \dot{s}\left(t_{0}\right) .
$$

Since $\dot{s}(t)>0$ via Lemma 2.1 , it follows that

$$
v\left(s\left(t_{0}\right), t_{0}\right)>\mu \text {. }
$$

If the minimum occurs on $t=0$, then by assumption $\mathrm{A}$,

$$
0<\psi_{*} \leq v(x, t) .
$$

Combining (2.14) and (2.15) we obtain

$$
0<\min \left(\mu, \psi_{*}\right) \leq v(x, t)
$$

for $0 \leq x \leq s(t)$ and $0 \leq t \leq T$. 
We turn now to the estimation of an upper bound for $v$. Set $v=w+z$, where

$$
\begin{array}{ll}
w_{t}=\beta w_{x x}, & 0<x<s(t), 0<t \leq T, \\
w(x, 0)=\psi(x), & 0 \leq x \leq b=s(0), \\
w_{x}(0, t)=0, & 0<t \leq T, \\
\beta w_{x}(s(t), t)=(\mu-v(s(t), t)) \dot{s}(t), & 0<t \leq T,
\end{array}
$$

and

$$
\begin{array}{ll}
z_{t}=\beta w_{x x}, & 0<x<s(t), 0<t \leq T, \\
z(x, 0)=0, & 0 \leq x \leq b=s(0), \\
-\beta z_{x}(0, t)=g(t), & 0<t \leq T, \\
\beta z_{x}(s(t), t)=0, & 0<t \leq T .
\end{array}
$$

The estimate of $z$ follows in a manner similar to the $z$ of $(2.2)$. Thus, we see that

$$
0 \leq z(x, t) \leq\left(b^{-1} T+(2 \beta b)^{-1}\right) g^{*} .
$$

Considering $w$, we see from the maximum principle that the maximum value for $w$ on $0 \leq x \leq s(t), 0 \leq t \leq T$, must occur on $x=s(t)$ or $t=0$. If it occurs on $x=s(t)$ at $\left(s\left(t_{0}\right), t_{0}\right)$, then the parabolic version of Hopf's lemma implies that

$$
0<\beta w_{x}\left(s\left(t_{0}\right), t_{0}\right)=\left(\mu-v\left(s\left(t_{0}\right), t_{0}\right)\right) \dot{s}\left(t_{0}\right) .
$$

Since $\dot{s}(t)>0$,

$$
v\left(s\left(t_{0}\right), t_{0}\right)<\mu \text {. }
$$

Since $z \geq 0$,

$$
w(x, t) \leq w\left(s\left(t_{0}\right), t_{0}\right) \leq w\left(s\left(t_{0}\right), t_{0}\right)+z\left(s\left(t_{0}\right), t_{0}\right)=v\left(s\left(t_{0}\right), t_{0}\right)<\mu .
$$

If the maximum value of $w$ occurs on $t=0$, then

$$
w(x, t) \leq \psi^{*} \text {. }
$$

Combining (2.16), (2.19), (2.22), and (2.23) we can state the following estimate.

Lemma 2.3. For $0 \leq x \leq s(t), 0 \leq t \leq T$,

$$
0<v_{*}=\min \left(\mu, \psi_{*}\right) \leq v(x, t) \leq \max \left(\mu, \psi^{*}\right)+\left(b^{-1} T+(2 \beta b)^{-1}\right) g^{*} .
$$

Proof. See the analysis preceding the statement of the lemma.

Combining Lemma 2.1 through Lemma 2.3 we obtain the following estimate.

LEMMA 2.4. For $0<t \leq T$,

$$
0<\dot{s}(t) \leq s^{*}=\nu\left(v^{*}\right) \exp \left(-\frac{\delta}{v^{*}}\right) F\left(u^{*}\right)
$$

where

$$
\left\{\begin{array}{l}
u^{*}=\phi^{*}+\left(b^{-1} T+(2 \alpha b)^{-1}\right) f^{*}, \\
v^{*}=\max \left(\mu, \psi^{*}\right)+\left(b^{-1} T+(2 \beta b)^{-1}\right) g^{*} \\
v_{*}=\min \left(\mu, \psi_{*}\right) .
\end{array}\right.
$$


Proof. This is a simple consequence of assumption $\mathrm{C}$ and the substitution of the estimates (2.12) and (2.24) into (1.2).

We can obtain another estimate by an elementary calculation.

LEMMA 2.5. For $0 \leq t \leq T$,

$$
b \leq s(t) \leq b+\gamma^{-1} \int_{0}^{b} \phi(x) d x+\gamma^{-1} \int_{0}^{t} f(\tau) d \tau .
$$

Proof. See the analysis preceding the statement of the lemma.

3. Existence of a solution. For each $\varepsilon>0$ we consider the problem

$$
\begin{array}{ll}
u_{t}^{\varepsilon}=\alpha u_{x x}^{\varepsilon}, & 0<x<s^{\varepsilon}(t), 0<t \leq T, \\
v_{t}^{\varepsilon}=\beta v_{x x}^{\varepsilon}, & 0<x<s^{\varepsilon}(t), 0<t \leq T, \\
u^{\varepsilon}(x, 0)=\phi(x), & 0<x<b=s^{\varepsilon}(0), \\
v^{\varepsilon}(x, 0)=\psi(x), & 0<x<b=s^{\varepsilon}(0), \\
-\alpha u_{x}^{\varepsilon}(0, t)=f(t), & 0<t \leq T, \\
-\beta v_{x}^{\varepsilon}(0, t)=g(t), & 0<t \leq T, \\
\alpha u_{x}^{\varepsilon}\left(s^{\varepsilon}(t), t\right)=-\left(\gamma+u^{\varepsilon}\left(s^{\varepsilon}(t), t\right)\right) \dot{s}^{\varepsilon}(t), & 0<t \leq T, \\
\beta v_{x}^{\varepsilon}\left(s^{\varepsilon}(t), t\right)=\left(\mu-v^{\varepsilon}\left(s^{\varepsilon}(t), t\right)\right) \dot{s}^{\varepsilon}(t), & 0<t \leq T,
\end{array}
$$

where

$$
\left\{\begin{array}{l}
\dot{s}^{\varepsilon}(t)=\max \left\{\nu\left(\bar{v}^{\varepsilon}(t)\right) \exp \left\{-\frac{\delta}{\bar{v}^{\varepsilon}(t)}\right\} F\left(\bar{u}^{\varepsilon}(t)\right), 0\right\}, \quad 0<t \leq T \\
s^{\varepsilon}(0)=b
\end{array}\right.
$$

and where

$$
\bar{u}^{\varepsilon}(t)= \begin{cases}\phi(b), & 0 \leq t \leq \varepsilon \\ u^{\varepsilon}\left(s^{\varepsilon}(t-\varepsilon), t-\varepsilon\right), & \varepsilon \leq t \leq T\end{cases}
$$

and

$$
\bar{v}^{\varepsilon}(t)= \begin{cases}\psi(b), & 0 \leq t \leq \varepsilon \\ v^{\varepsilon}\left(s^{\varepsilon}(t-\varepsilon), t-\varepsilon\right), & \varepsilon \leq t \leq T\end{cases}
$$

Since the differential equation (3.2) is independent of the solution for $0 \leq t \leq \varepsilon$, we note that $s^{\varepsilon}(t)$ is determined in this interval and that problem (3.1) can be solved uniquely for the bounded solution using classical means [C] for the domain $0 \leq x \leq s^{\varepsilon}(t), 0 \leq t \leq \varepsilon$. Since this determines $\bar{u}^{\varepsilon}(t)$ and $\bar{v}^{\varepsilon}(t)$ for $\varepsilon \leq t \leq 2 \varepsilon$, from whence $s^{\varepsilon}(t)$ is determined, we see that the bounded solution of (3.1) can be carried out in the domain $0 \leq x \leq s^{\varepsilon}(t)$, $\varepsilon \leq t \leq 2 \varepsilon$ using $u^{\varepsilon}(x, \varepsilon)$ and $v^{\varepsilon}(x, \varepsilon)$ as initial conditions. Clearly, this process can be continued to produce a unique solution to problem (3.1). 
Arguing as in Sec. 2, we find the following estimates:

$$
\begin{aligned}
-\gamma & \leq u^{\varepsilon}(x, t) \leq u^{*}, & & 0 \leq x \leq s^{\varepsilon}(t), 0 \leq t \leq T, \\
0 & \leq v^{\varepsilon}(x, t) \leq v^{*}, & & 0 \leq x \leq s^{\varepsilon}(t), 0 \leq t \leq T, \\
0 & <v_{*} \leq v^{\varepsilon}\left(s^{\varepsilon}(t), t\right), & & 0 \leq t \leq T,
\end{aligned}
$$

and

$$
0<\dot{s}^{\varepsilon}(t) \leq s^{*}, \quad 0 \leq t \leq T .
$$

We note that these estimates are independent of $\varepsilon$. From the estimates (3.5)-(3.8) via the interior Schauder estimates [C, FR] we obtain the equiboundedness of $u_{t}^{\varepsilon}, u_{x t}^{\varepsilon}, u_{x x t}^{\varepsilon}, u_{t t}^{\varepsilon}$, $v_{t}^{\varepsilon}, v_{x t}^{\varepsilon}, v_{x x t}^{\varepsilon}$, and $v_{t t}^{\varepsilon}$ on the sets $0<\rho \leq x \leq s(t)-\rho, \rho \leq t \leq t$ for each $\rho>0$. We especially note from the integral representation of the solution $[\mathrm{C}]$ that

$$
\left|u_{x}^{\varepsilon}(x, t)\right|,\left|v_{x}^{\varepsilon}(x, t)\right| \leq C\left(1+\frac{1}{\sqrt{t}}\right), \quad 0 \leq x \leq s^{\varepsilon}(t), \quad 0<t \leq T,
$$

where the constant $C$ is independent of $\varepsilon$.

We also need the equicontinuity of $u^{\varepsilon}\left(s^{\varepsilon}(t), t\right)$ and $v^{\varepsilon}\left(s^{\varepsilon}(t), t\right)$ in order to demonstrate the existence of the solution. Now for $0<\rho \leq t_{1} \leq t_{2} \leq T$, and $d>0$, we use the mean value theorem to obtain

$$
\begin{aligned}
& u^{\varepsilon}\left(s^{\varepsilon}\left(t_{2}\right), t_{2}\right)-u^{\varepsilon}\left(s^{\varepsilon}\left(t_{1}\right), t_{1}\right)=\left\{u^{\varepsilon}\left(s^{\varepsilon}\left(t_{2}\right), t_{2}\right)-u^{\varepsilon}\left(s^{\varepsilon}\left(t_{2}\right)-d, t_{2}\right)\right\} \\
&+\left\{u^{\varepsilon}\left(s^{\varepsilon}\left(t_{2}\right)-d, t_{2}\right)-u^{\varepsilon}\left(s^{\varepsilon}\left(t_{2}\right)-d, t_{1}\right)\right\} \\
&+\left\{u^{\varepsilon}\left(s^{\varepsilon}\left(t_{2}\right)-d, t_{1}\right)-u^{\varepsilon}\left(s^{\varepsilon}\left(t_{1}\right)-d, t_{1}\right)\right\} \\
&+\left\{u^{\varepsilon}\left(s^{\varepsilon}\left(t_{1}\right)-d, t_{1}\right)-u^{\varepsilon}\left(s^{\varepsilon}\left(t_{1}\right), t_{1}\right)\right\} \\
&= u_{x}^{\varepsilon}\left(\xi_{1}, t_{2}\right) d+u_{t}^{\varepsilon}\left(s^{\varepsilon}\left(t_{2}\right)-d, \bar{t}\right)\left(t_{2}-t_{1}\right) \\
&+u_{x}^{\varepsilon}\left(\xi_{2}, t_{1}\right) \dot{s}^{\varepsilon}(\tilde{t})\left(t_{2}-t_{1}\right)+u_{x}^{\varepsilon}\left(\xi_{3}, t_{1}\right)(-d) .
\end{aligned}
$$

From Lemma 2.1 and Lemma 2.2 and the interior Schauder estimates [C, FR] we obtain the existence of a positive constant $\mu_{\rho}$ which is independent of $\varepsilon$, so that

$$
\left|u^{\varepsilon}\left(s^{\varepsilon}\left(t_{2}\right), t_{2}\right)-u^{\varepsilon}\left(s^{\varepsilon}\left(t_{1}\right), t_{1}\right)\right| \leq \mu_{\rho}\left(d+\left(t_{2}-t_{1}\right) d^{-3}+\left(t_{2}-t_{1}\right)\right) .
$$

Selecting $d=\left(t_{2}-t_{1}\right)^{1 / 4}$ we see that

$$
\left|u^{\varepsilon}\left(s^{\varepsilon}\left(t_{2}\right), t_{2}\right)-u^{\varepsilon}\left(s^{\varepsilon}\left(t_{1}\right), t_{1}\right)\right| \leq 3 \mu_{\rho}\left(t_{2}-t_{1}\right)^{1 / 4}
$$

holds for $0<\rho \leq t_{1} \leq t_{2} \leq T$. Similarly, we have

$$
\left|v^{\varepsilon}\left(s^{\varepsilon}\left(t_{2}\right), t_{2}\right)-v^{\varepsilon}\left(s^{\varepsilon}\left(t_{1}\right), t_{1}\right)\right| \leq 3 \mu_{\rho}\left(t_{2}-t_{1}\right)^{1 / 4},
$$

for $0<\rho \leq t_{1} \leq t_{2} \leq T$.

Applying the Cantor diagonalization process, we can find a sequence $\varepsilon_{n} \rightarrow 0$ and functions $u(x, t), v(x, t), s(t), \bar{u}(t)$, and $\bar{v}(t)$ such that

$$
\begin{gathered}
\left\{\begin{array}{l}
u(x, t)=\lim _{n \rightarrow \infty} u^{\varepsilon_{n}}(x, t), \quad \rho \leq x \leq s(t), \rho \leq t \leq T, \\
v(x, t)=\lim _{n \rightarrow \infty} v^{\varepsilon_{n}}(x, t), \quad \rho \leq x \leq s(t), \rho \leq t \leq T,
\end{array}\right. \\
\begin{cases}s(t)=\lim _{n \rightarrow \infty} s^{\varepsilon_{n}}(t), \quad \rho \leq t \leq T, \\
\dot{s}(t)=\lim _{n \rightarrow \infty} \dot{s}^{\varepsilon_{n}}(t), \quad \rho \leq t \leq T,\end{cases}
\end{gathered}
$$


and

$$
\begin{cases}\bar{u}(t)=\lim _{n \rightarrow \infty} u^{\varepsilon_{n}}\left(s^{\varepsilon_{n}}(t), t\right), & \rho \leq t \leq T, \\ \bar{v}(t)=\lim _{n \rightarrow \infty} v^{\varepsilon_{n}}\left(s^{\varepsilon_{n}}(t), t\right), & \rho \leq t \leq T .\end{cases}
$$

The convergence in each of (3.14)-(3.16) is uniform for $\rho>0$. Moreover, the convergences of $u_{x x}^{\varepsilon_{n}}, u_{t}^{\varepsilon_{n}}, v_{x x}^{\varepsilon_{n}}$, and $v_{t}^{\varepsilon_{n}}$ imply that the limit functions $u(x, t)$ and $v(x, t)$ satisfy $u_{t}=\alpha u_{x x}$ and $v_{t}=\beta v_{x x}$ for $0<x<s(t), 0<t \leq T$.

Now we show that the triple $(u, v, s)$ satisfies our problem (1.1)-(1.2). First we show that

$$
\begin{cases}\bar{u}(t)=\lim _{x \rightarrow s(t)} u(x, t) & \text { uniformly in } \rho \leq t \leq T, \\ \bar{v}(t)=\lim _{x \rightarrow s(t)} v(x, t) & \text { uniformly in } \rho \leq t \leq T\end{cases}
$$

for each $\rho>0$. Since

$$
\bar{u}(t)-u(x, t)=\left\{\bar{u}(t)-u^{\varepsilon_{n}}\left(s^{\varepsilon_{n}}(t), t\right)\right\}+\left\{u^{\varepsilon_{n}}\left(s^{\varepsilon_{n}}(t), t\right)-u^{\varepsilon_{n}}(x, t)\right\}+\left\{u^{\varepsilon_{n}}(x, t)-u(x, t)\right\},
$$

it follows from (3.9) that

$$
\begin{aligned}
|\bar{u}(t)-u(x, t)| \leq \mid & \bar{u}(t)-u^{\varepsilon_{n}}\left(s^{\varepsilon_{n}}(t), t\right) \mid \\
& +\mu_{\rho}\left(s^{\varepsilon_{n}}(t)-x\right)+\left|u^{\varepsilon_{n}}(x, t)-u(x, t)\right| .
\end{aligned}
$$

Letting $n \rightarrow \infty$, we obtain

$$
|\bar{u}(t)-u(x, t)| \leq \mu_{\rho}|s(t)-x|
$$

for each $x, \rho \leq x \leq s(t), \rho \leq t \leq T$. Consequently, we have

$$
\bar{u}(t)=u(s(t), t) .
$$

Moreover, $u(x, t)$ is continuous at $(s(t), t)$, since

$$
\begin{aligned}
|u(x, \tau)-\bar{u}(t)| & \leq|u(x, \tau)-\bar{u}(\tau)|+|\bar{u}(\tau)-\bar{u}(t)| \\
& \leq \mu_{\rho}\left\{|x-s(t)|+|t-\tau|^{1 / 4}\right\}
\end{aligned}
$$

for each $\rho>0, \rho \leq x \leq s(t), \rho \leq t \leq T$. For the same reason we have $\bar{v}(t)=v(s(t), t)$, and $v(x, t)$ is continuous at $(s(t), t)$. Hence (3.17) holds. Thus, we see from the uniform convergence of $\dot{s}^{\varepsilon_{n}}(t)=\max \left\{\nu\left(\bar{v}^{\varepsilon_{n}}(t)\right) \exp \left\{-\frac{\delta}{\bar{v}^{\varepsilon_{n}}}\right\} F\left(\bar{u}^{\varepsilon_{n}}(t)\right), 0\right\}$ that the triple $(u, v, s)$ satisfies (1.2). It remains to show that $(u, v, s)$ satisfies the initial-boundary conditions and the indicated continuity in the definition of solutions.

From [C] we can represent $u^{\varepsilon_{n}}(x, t)$ and $v^{\varepsilon_{n}}(x, t)$ and $v^{\varepsilon_{n}}(x, t)$ in $0<x<b, 0<t \leq T$ as

$$
\begin{aligned}
u^{\varepsilon_{n}}(x, t)=\int_{0}^{b} & {\left[\theta\left((x-\xi) b^{-1}, \alpha b^{-2} t\right)+\theta\left((x+\xi) b^{-1}, \alpha b^{-2} t\right)\right] \phi(\xi) d \xi } \\
& +2 \alpha b^{-1} \int_{0}^{t} \theta\left(x b^{-1}, \alpha b^{-2}(t-\tau)\right) f(\tau) d \tau \\
& +2 \alpha b^{-1} \int_{0}^{t} \theta\left((x-b) b^{-1}, \alpha b^{-2}(t-\tau)\right) u_{x}^{\varepsilon_{n}}(b, \tau) d \tau
\end{aligned}
$$


and

$$
\begin{aligned}
v^{\varepsilon_{n}}(x, t)=\int_{0}^{b} & {\left[\theta\left((x-\xi) b^{-1}, \beta b^{-2} t\right)+\theta\left((x+\xi) b^{-1}, \beta b^{-2} t\right)\right] \psi(\xi) d \xi } \\
& +2 \beta b^{-1} \int_{0}^{t} \theta\left(x b^{-1}, \beta b^{-2}(t-\tau)\right) g(\tau) d \tau \\
& +2 \beta b^{-1} \int_{0}^{t} \theta\left((x-b) b^{-1}, \beta b^{-2}(t-\tau)\right) v_{x}^{\varepsilon_{n}}(b, \tau) d \tau
\end{aligned}
$$

where

$$
\theta(x, t)=\sum_{m=-\infty}^{\infty} K(x+2 m, t), \quad t>0
$$

and

$$
K(x, t)=\frac{1}{\sqrt{4 \pi t}} \exp \left\{-\frac{x^{2}}{4 t}\right\}, \quad t>0
$$

By the Lebesgue dominated convergence theorem, letting $\varepsilon_{n} \rightarrow 0$ in (3.22)-(3.23) shows that $u=u(x, t)$ and $v=v(x, t)$ satisfy (3.22)-(3.23) with $\varepsilon_{n}$ removed. Hence we obtain

$$
\begin{cases}-\alpha u_{x}(0, t)=f(t), \quad-\beta v_{x}(0, t)=g(t), & 0<t \leq T, \\ u(x, 0)=\phi(x), \quad v(x, 0)=\psi(x), & 0<x<b .\end{cases}
$$

Hence, by $[\mathrm{C}]$, we see that $u(x, t)$ and $v(x, t)$ satisfy the continuity conditions in the definition of a solution.

Now for each $\varepsilon>0$ let

$$
x=s^{\varepsilon}(t)+\rho \eta-\rho,
$$

with $\rho>0$ and small, and

$$
\left\{\begin{array}{l}
w^{\varepsilon}(\eta, t)=u^{\varepsilon}\left(s^{\varepsilon}(t)+\rho \eta-\rho, t\right) \\
z^{\varepsilon}(\eta, t)=v^{\varepsilon}\left(s^{\varepsilon}(t)+\rho \eta-\rho, t\right)
\end{array}\right.
$$

Then $w^{\varepsilon}(\eta, t)$ and $z^{\varepsilon}(\eta, t)$ satisfy

$$
\begin{array}{ll}
w_{t}^{\varepsilon}=\alpha \rho^{-2} w_{\eta \eta}^{\varepsilon}+\dot{s}^{\varepsilon}(t) \rho^{-1} w_{\eta}^{\varepsilon}, & 0<\eta<1,0<t \leq T, \\
z_{t}^{\varepsilon}=\beta \rho^{-2} z_{\eta \eta}^{\varepsilon}+\dot{s}^{\varepsilon}(t) \rho^{-1} z_{\eta}^{\varepsilon}, & 0<\eta<1,0<t \leq T, \\
w^{\varepsilon}(\eta, 0)=\phi(b+\rho \eta-\rho), & 0<\eta<1, \\
z^{\varepsilon}(\eta, 0)=\psi(b+\rho \eta-\rho), & 0<\eta<1, \\
w_{\eta}^{\varepsilon}(0, t)=\rho u_{x}^{\varepsilon}\left(s^{\varepsilon}(t)-\rho, t\right), & 0<t \leq T, \\
z_{\eta}^{\varepsilon}(0, t)=\rho u_{x}^{\varepsilon}\left(s^{\varepsilon}(t)-\rho, t\right), & 0<t \leq T, \\
w_{\eta}^{\varepsilon}(1, t)=-\alpha^{-1} \rho\left(\gamma+w^{\varepsilon}(1, t)\right) \dot{s}^{\varepsilon}(t), & 0<t \leq T, \\
z_{\eta}^{\varepsilon}(1, t)=\beta^{-1} \rho\left(\mu-z^{\varepsilon}(1, t)\right) \dot{s}^{\varepsilon}(t), & 0<t \leq T .
\end{array}
$$


Now we can represent $w^{\varepsilon}(\eta, t)$ and $z^{\varepsilon}(\eta, t)$ as

$$
\begin{aligned}
w^{\varepsilon}(\eta, t)=\int_{0}^{1} & {\left[\theta\left(\eta-\xi, \alpha \rho^{-2} t\right)+\theta\left(\eta+\xi, \alpha \rho^{-2} t\right)\right] \phi(b+\rho \xi-\rho) d \xi } \\
& -2 \int_{0}^{t} \theta\left(\eta, \alpha \rho^{-2}(t-\tau)\right) w_{\eta}^{\varepsilon}(0, \tau) d \tau \\
& +2 \int_{0}^{t} \theta\left(\eta-1, \alpha \rho^{-2}(t-\tau)\right)\left[-\alpha^{-1} \rho\left(\gamma+w^{\varepsilon}(1, \tau)\right) \dot{s}^{\varepsilon}(\tau)\right] d \tau \\
& +\int_{0}^{t} \int_{0}^{1}\left[\theta\left(\eta-\xi, \alpha \rho^{-2}(t-\tau)\right)+\theta\left(\eta+\xi, \alpha \rho^{-2}(t-\tau)\right)\right] \dot{s}^{\varepsilon}(\tau) w_{\xi}^{\varepsilon}(\xi, \tau) d \xi d \tau
\end{aligned}
$$

and

$$
\begin{aligned}
z^{\varepsilon}(\eta, t)= & \left.\int_{0}^{1} \theta\left(\eta-\xi, \beta \rho^{-2} t\right)+\theta\left(\eta+\xi, \beta \rho^{-2} t\right)\right] \psi(b+\rho \xi-\rho) d \xi \\
& -2 \int_{0}^{t} \theta\left(\eta, \beta \rho^{-2}(t-\tau)\right) z_{\eta}^{\varepsilon}(0, \tau) d \tau \\
& +2 \int_{0}^{t} \theta\left(\eta-1, \beta \rho^{-2}(t-\tau)\right)\left[\beta^{-1} \rho\left(u-z^{\varepsilon}(1, \tau)\right) \dot{s}^{\varepsilon}(\tau)\right] d \tau \\
& +\int_{0}^{t} \int_{0}^{1}\left[\theta\left(\eta-\xi, \beta \rho^{-2}(t-\tau)\right)+\theta\left(\eta+\xi, \beta \rho^{-2}(t-\tau)\right)\right] \dot{s}^{\varepsilon}(\tau) z_{\xi}^{\varepsilon}(\xi, \tau) d \xi d \tau .
\end{aligned}
$$

From (3.9), we see that there is a positive constant $C$ independent of $\varepsilon$ such that

$$
\left|w_{\xi}^{\varepsilon}(\xi, t)\right|,\left|z_{\xi}^{\varepsilon}(\xi, t)\right| \leq C\left(1+t^{-1 / 2}\right), \quad 0 \leq \xi \leq 1, \quad 0<t \leq T .
$$

By the convergence of $u_{t}^{\varepsilon_{n}}, u_{x x}^{\varepsilon_{n}}, u_{x}^{\varepsilon_{n}}, v_{t}^{\varepsilon_{n}}, v_{x x}^{\varepsilon_{n}}, v_{x}^{\varepsilon_{n}}$, and $s^{\varepsilon_{n}}, \dot{s}^{\varepsilon}$, we obtain the convergence of $w_{t}^{\varepsilon_{n}}, w_{\eta \eta}^{\varepsilon_{n}}, w_{\eta}^{\varepsilon_{n}}, z_{t}^{\varepsilon_{n}}, z_{\eta \eta}^{\varepsilon_{n}}$, and $z_{\eta}^{\varepsilon_{n}}$. Furthermore, it follows from the Lebesgue dominated convergence theorem and (3.32) that the limit functions $w(\eta, t)$ and $z(\eta, t)$ satisfy (3.29)(3.31) with $\varepsilon$ removed and

$$
\left\{\begin{array}{l}
w(\eta, t)=u(s(t)+\rho \eta-\rho, t), \\
z(\eta, t)=v(s(t)+\rho \eta-\rho, t)
\end{array}\right.
$$

and

$$
\left\{\begin{array}{l}
w_{\eta}(1, t)=-\alpha^{-1} \rho(\gamma+w(1, t)) \dot{s}(t), \\
z_{\eta}(1, t)=\beta^{-1} \rho(\mu-z(1, t)) \dot{s}(t),
\end{array}\right.
$$

where $u, v$ and $s, \dot{s}$ are the limit functions of $u^{\varepsilon}, v^{\varepsilon}$ and $s^{\varepsilon}, \dot{s}^{\varepsilon}$. Hence under the transforms

$$
u(x, t)=w\left(\frac{x+\rho-s(t)}{\rho}, t\right),
$$

and

$$
v(x, t)=z\left(\frac{x+\rho-s(t)}{\rho}, t\right),
$$

we see that $u$ and $v$ satisfy $(1.1 \mathrm{~g})-(1.1 \mathrm{~h})$. This completes the proof of the existence of a solution. 
4. Uniqueness and continuous dependence. In order to establish uniqueness and continuous dependence upon the data it will be convenient for us to introduce the change of independent variable $x=\xi s(t)$ and dependent variables $U(\xi, t)=u(\xi s(t), t)$, and $V(\xi, t)=v(\xi s(t), t)$. Then we can rewrite problem (1.1)-(1.2) as

$$
\begin{array}{ll}
U_{t}=\frac{\alpha}{s^{2}(t)} U_{\xi \xi}+\xi \frac{\dot{s}(t)}{s(t)} U_{\xi}, & 0<\xi<1,0<t \leq T, \\
V_{t}=\frac{\alpha}{s^{2}(t)} V_{\xi \xi}+\xi \frac{\dot{s}(t)}{s(t)} V_{\xi}, & 0<\xi<1,0<t \leq T, \\
U(\xi, 0)=\phi(\xi b), & 0 \leq \xi \leq 1, \\
V(\xi, 0)=\psi(\xi b), & 0 \leq \xi \leq 1, \\
-\alpha U_{\xi}(0, t)=f(t) s(t), & 0<t \leq T, \\
-\beta V_{\xi}(0, t)=g(t) s(t), & 0<t \leq T, \\
\alpha U_{\xi}(1, t)=-(\gamma+U(1, t)) s(t) \dot{s}(t), & 0<t \leq T, \\
\beta V_{\xi}(1, t)=(\mu-V(1, t)) s(t) \dot{s}(t), & 0<t \leq T,
\end{array}
$$

and

$$
\left\{\begin{array}{l}
\dot{s}(t)=\nu(V(1, t)) \exp \left\{-\frac{\delta}{V(1, t)}\right\} F(U(1, t)), \quad 0<t \leq T \\
s(0)=b
\end{array}\right.
$$

Now suppose that $\left(U_{1}, V_{1}, s_{1}\right)$ and $\left(U_{2}, V_{2}, s_{2}\right)$ denote solutions to (4.1)-(4.2) with data $\phi_{i}, \psi_{i}, f_{i}, g_{i}, \nu_{i}, \delta_{i}, F_{i}, \gamma_{i}, \mu_{i}$, and $b_{i}, i=1,2$, respectively. Let $W=U_{1}-U_{2}$ and $Z=V_{1}-V_{2}$. We note that the $s_{i}, i=1,2$, are determined by (4.2). Consequently it suffices to estimate $W$ and $Z$ to obtain estimates for $s_{1}-s_{2}$ and $\dot{s}_{1}-\dot{s}_{2}$. We assume that the data $\phi_{i}, \psi_{i}, f_{i}, g_{i}, \nu_{i}, F_{i}$, and $b_{i}$ satisfy the bounds in assumptions $\mathrm{A}, \mathrm{B}$, and $\mathrm{C}$, which implies that $u_{i}, v_{i}$, and $s_{i}, i=1,2$, satisfy the a priori bounds obtained in Sec. 2 . Differencing the equations for $\left(U_{i}, V_{i}, s_{i}\right), i=1,2$, we see that $W$ and $Z$ satisfy

$$
\begin{array}{ll}
W_{t}=\frac{\alpha}{s_{1}^{2}} U_{1, \xi \xi}-\frac{\alpha}{s_{2}^{2}} U_{2, \xi \xi}+\xi \frac{\dot{s}_{1}}{s_{1}} U_{1, \xi}-\xi \frac{\dot{s}_{2}}{s_{2}} U_{2, \xi}, & 0<\xi<1,0<t \leq T, \\
Z_{t}=\frac{\beta}{s_{1}^{2}} V_{1, \xi \xi}-\frac{\beta}{s_{2}^{2}} V_{2, \xi \xi}+\xi \frac{\dot{s}_{1}}{s_{1}} V_{1, \xi}-\xi \frac{\dot{s}_{2}}{s_{2}} V_{2, \xi}, & 0<\xi<1,0<t \leq T, \\
W(0, t)=\phi_{1}\left(b_{1} \xi\right)-\phi_{2}\left(b_{2} \xi\right), & 0<t \leq T, \\
Z(0, t)=\psi_{1}\left(b_{1} \xi\right)-\psi_{2}\left(b_{2} \xi\right), & 0<t \leq T, \\
-\alpha W_{\xi}(0, t)=f_{1}(t) s_{1}(t)-f_{2}(t) s_{2}(t), & 0<t \leq T, \\
-\beta Z_{\xi}(0, t)=g_{1}(t) s_{1}(t)-g_{2}(t) s_{2}(t), & 0<t \leq T, \\
\alpha W_{\xi}(1, t)=-\left(\gamma_{1} s_{1}(t) \dot{s}_{1}(t)-\gamma_{2} s_{2}(t) \dot{s}_{2}(t)\right) & \\
\quad-\left(U_{1}(1, t) s_{1}(t) \dot{s}_{1}(t)-U_{2}(1, t) s_{2}(t) \dot{s}_{2}(t)\right), & 0<t \leq T, \\
\beta Z_{\xi}(1, t)=+\left(\mu_{1} s_{1}(t) \dot{s}_{1}(t)-\mu_{2} s_{2}(t) \dot{s}_{2}(t)\right) & \\
\quad-\left(V_{1}(1, t) s_{1}(t) \dot{s}_{1}(t)-V_{2}(1, t) s_{2}(t) \dot{s}_{2}(t)\right), & 0<t \leq T,
\end{array}
$$


and

$$
\left\{\begin{aligned}
\dot{s}_{1}(t)-\dot{s}_{2}(t)= & \nu_{1}\left(V_{1}(1, t) \exp \left\{-\frac{\delta_{1}}{V_{1}(1, t)}\right\} F\left(U_{1}(1, t)\right)\right. \\
& \quad-\nu_{2}\left(V_{2}(1, t) \exp \left\{-\frac{\delta_{2}}{V_{2}(1, t)}\right\} F\left(U_{2}(1, t)\right)\right. \\
s_{1}(0)-s_{2}(0)= & b_{1}-b_{2} .
\end{aligned}\right.
$$

Consider first the equation for $W$. By elementary calculations we see that

$$
W_{t}=\frac{\alpha_{1}}{s_{1}^{2}(t)} W_{\xi \xi}+\xi \frac{\dot{s}_{1}(t)}{s_{1}(t)} W_{\xi}+\left(\frac{\alpha}{s_{1}^{2}(t)}-\frac{\alpha}{s_{2}^{2}(t)}\right) U_{2, \xi \xi}+\xi\left(\frac{\dot{s}_{1}(t)}{s_{1}(t)}-\frac{\dot{s}_{2}(t)}{s_{2}(t)}\right) U_{2, \xi}
$$

Multiplying by $W$ and integrating with respect to $\xi$ from 0 to 1 we obtain

$$
\begin{aligned}
\frac{1}{2} \frac{d}{d t} \int_{0}^{1} W^{2}(\xi, t) d \xi+\frac{\alpha_{1}}{s_{1}^{2}(t)} \int_{0}^{1} W_{\xi}^{2}(\xi, t) d \xi & =\frac{\alpha_{1}}{s_{1}^{2}(t)}\left[W(1, t) W_{\xi}(1, t)-W(0, t) W_{\xi}(0, t)\right] \\
& +\xi \frac{\dot{s}_{1}(t)}{s_{1}(t)} \int_{0}^{1} W(\xi, t) W_{\xi}(\xi, t) d \xi \\
& +\left(\frac{\alpha_{1}}{s_{1}^{2}(t)}-\frac{\alpha_{2}}{s_{2}^{2}(t)}\right)\left[W(1, t) U_{2, \xi}(1, t)-W(0, t) U_{2, \xi}(0, t)\right. \\
& +\left(\frac{\dot{s}_{1}(t)}{s_{1}(t)}-\frac{\dot{s}_{2}(t)}{s_{2}(t)}\right) \int_{0}^{1} \xi W(\xi, t) U_{2, \xi}(\xi, t) d \xi \\
=I_{1} & +I_{2}+I_{3}+I_{4}+I_{5} .
\end{aligned}
$$

We shall denote all constants in what follows as $C$ when they depend only on the data and $T$. Those constants that depend on a parameter $\varepsilon$ will be denoted by $C(\varepsilon)$.

From the equation

$$
W^{2}(1, t)=\int_{0}^{1} W^{2}(\xi, t) d \xi+2 \int_{0}^{1} \xi W(\xi, t) W_{\xi}(\xi, t) d \xi
$$

and the inequality

$$
a b \leq \frac{1}{4 \varepsilon} a^{2}+\varepsilon b^{2},
$$

we obtain

$$
W^{2}(1, t) \leq C(\varepsilon) \int_{0}^{1} W^{2}(\xi, t) d \xi+\varepsilon \int_{0}^{1} W_{\xi}^{2}(\xi, t) d \xi
$$

and similarly

$$
W^{2}(0, t) \leq C(\varepsilon) \int_{0}^{1} W^{2}(\xi, t) d \xi+\varepsilon \int_{0}^{1} W_{\xi}^{2}(\xi, t) d \xi
$$


Consider $I_{1}$. First,

$$
\begin{aligned}
\left|W(1, t) W_{\xi}(1, t)\right| \leq & \frac{1}{\alpha}|W(1, t)|\left(\left|\gamma_{1} s_{1} \dot{s}_{1}-\gamma_{2} s_{2} \dot{s}_{2}\right|+\left|U_{1}(1, t) s_{1} \dot{s}_{1}-U_{2}(1, t) s_{2} \dot{s}_{2}\right|\right) \\
\leq & \frac{1}{\alpha}|W(1, t)|\left(\left|\gamma_{1}-\gamma_{2}\right|\left|s_{1} \dot{s}_{1}\right|+\left|s_{1}-s_{2}\right|\left|\gamma_{2} \dot{s}_{1}\right|+\left|\dot{s}_{1}-\dot{s}_{2}\right|\left|s_{2} \gamma_{2}\right|\right) \\
& \quad+\frac{1}{\alpha}|W(1, t)|\left(|W(1, t)|\left|s_{1} \dot{s}_{1}\right|+\left|U_{2}(1, t) \dot{s}_{1}\right|\left|s_{1}-s_{2}\right|\right. \\
& \left.\quad+\left|U_{2}(1, t) s_{2}\right||| \dot{s}_{1}-\dot{s}_{2} \mid\right) \\
\leq & C\left(|W(1, t)|^{2}+|W(1, t)|\left|\gamma_{1}-\gamma_{2}\right|+|W(1, t)|\left|s_{1}-s_{2}\right|\right. \\
& \left.\quad+|W(1, t)|\left|\dot{s}_{1}-\dot{s}_{2}\right|\right),
\end{aligned}
$$

where we have used the a priori bounds on $u_{i}, v_{i}, s_{i}$, and $\dot{s}_{i}$ obtained in Sec. 2 . Since

$$
\begin{aligned}
\left|\dot{s}_{1}-\dot{s}_{2}\right| & \leq\left|\nu_{1}\left(V_{1}\right) \exp \left(-\frac{\delta_{1}}{V_{1}}\right) F_{1}\left(U_{1}\right)-\nu_{2}\left(V_{2}\right) \exp \left(-\frac{\delta_{2}}{V_{2}}\right) F_{2}\left(U_{2}\right)\right| \\
& \leq C\left\{|W(1, t)|+|Z(1, t)|+\left|\delta_{1}-\delta_{2}\right|+\left\|\nu_{1}-\nu_{2}\right\|_{v^{*}}+\left\|F_{1}-F_{2}\right\|_{u^{*}}\right\}
\end{aligned}
$$

where $\left\|\nu_{1}-\nu_{2}\right\|_{v^{*}}=\sup _{0 \leq v \leq v^{*}}\left|\nu_{1}(v)-\nu_{2}(v)\right|$ and $\left\|F_{1}-F_{2}\right\|_{u^{*}}=\sup _{0 \leq u \leq u^{*}} \mid F_{1}(u)-$ $F_{2}(u) \mid$, and

$$
\begin{aligned}
\left|s_{1}-s_{2}\right| \leq\left|b_{1}-b_{2}\right| & +\int_{0}^{t}\left|\dot{s}_{1}(\tau)-\dot{s}_{2}(\tau)\right| d \tau \\
\leq\left|b_{1}-b_{2}\right|+C\left\{\left|\delta_{1}-\delta_{2}\right|\right. & +\left\|\nu_{1}-\nu_{2}\right\|_{v^{*}}+\left\|F_{1}-F_{2}\right\|_{u^{*}} \\
& \left.+\int_{0}^{t}|W(1, \tau)| d \tau+\int_{0}^{t}|Z(1, \tau)| d \tau\right\},
\end{aligned}
$$

we obtain

$$
\begin{aligned}
\left|W(1, t) W_{\xi}(1, t)\right| \leq C\{ & |W(1, t)|^{2}+|W(1, t) Z(1, t)| \\
& +|W(1, t)| \int_{0}^{t}|W(1, \tau)| d \tau+|W(1, t)| \int_{0}^{t}|Z(1, \tau)| d \tau \\
& +\left|\gamma_{1}-\gamma_{2}\right|^{2}+\left|\delta_{1}-\delta_{2}\right|^{2}+\left|b_{1}-b_{2}\right|^{2}+\left\|\nu_{1}-\nu_{2}\right\|_{v^{*}}^{2} \\
& \left.+\left\|F_{1}-F_{2}\right\|_{u^{*}}^{2}\right\} .
\end{aligned}
$$

Now, applying the Schwarz inequality, we obtain

$$
\begin{aligned}
|W(1, t)| \int_{0}^{t}|W(1, \tau)| d \tau & \leq|W(1, t)| \sqrt{t}\left(\int_{0}^{t}|W(1, \tau)|^{2} d \tau\right)^{1 / 2} \\
& \leq C\left\{W^{2}(1, t)+\int_{0}^{t} W^{2}(1, \tau) d \tau\right\}
\end{aligned}
$$

and similarly,

$$
|W(1, t)| \int_{0}^{t}|Z(1, \tau)| d \tau \leq C\left\{W^{2}(1, t)+\int_{0}^{t} Z^{2}(1, \tau) d \tau\right\} .
$$


Hence, we obtain

$$
\begin{aligned}
\left|W(1, t) W_{\xi}(1, t)\right| \leq C & \left\{\left|\delta_{1}-\delta_{2}\right|^{2}+\left|b_{1}-b_{2}\right|^{2}+\left\|\nu_{1}-\nu_{2}\right\|_{v^{*}}+\left\|F_{1}-F_{2}\right\|_{u^{*}}\right. \\
+ & \left.W^{2}(1, t)+Z^{2}(1, t)+\int_{0}^{t} W^{2}(1, \tau) d \tau+\int_{0}^{t} Z^{2}(1, \tau) d \tau\right\} .
\end{aligned}
$$

In a similar manner we obtain

$$
\begin{aligned}
&\left|W(0, t) W_{\xi}(0, t)\right| \leq C|W(0, t)|\left\{\left\|f_{1}-f_{2}\right\|_{T}+\left|s_{1}(t)-s_{2}(t)\right|\right\} \\
& \leq C\left\{W^{2}(0, t)+\left\|f_{1}-f_{2}\right\|_{T}^{2}+\left|b_{1}-b_{2}\right|^{2}+\left|\delta_{1}-\delta_{2}\right|^{2}\right. \\
& \quad+\left\|\nu_{1}-\nu_{2}\right\|_{v^{*}}^{2}+\left\|F_{1}-F_{2}\right\|_{u^{*}}^{2} \\
&\left.\quad+\int_{0}^{t} W^{2}(1, \tau) d \tau+\int_{0}^{t} Z^{2}(1, \tau) d \tau\right\} .
\end{aligned}
$$

Hence, we have

$$
\begin{aligned}
\left|I_{1}\right| \leq C\{\mid & \delta_{1}-\left.\delta_{2}\right|^{2}+\left|b_{1}-b_{2}\right|^{2}+\left\|\nu_{1}-\nu_{2}\right\|_{v^{*}}^{2}+\left\|F_{1}-F_{2}\right\|_{u^{*}}^{2}+\left\|f_{1}-f_{2}\right\|_{T}^{2} \\
& \left.+W^{2}(1, t)+W^{2}(0, t)+Z^{2}(1, t)+\int_{0}^{t} W^{2}(1, \tau) d \tau+\int_{0}^{t} Z^{2}(1, \tau) d \tau\right\} .
\end{aligned}
$$

Clearly, applying inequality (4.8), we obtain

$$
\left|I_{2}\right| \leq C(\varepsilon) \int_{0}^{1} W^{2}(\xi, t) d \xi+\varepsilon \int_{0}^{1} W_{\xi}(\xi, t) d \xi
$$

Since the quantities $U_{2, \xi}(0, t)$, and $U_{2, \xi}(1, t)$ are bounded, as can be seen by a consideration of the data and the a priori estimates, and since

$$
\frac{\alpha}{s_{1}^{2}}-\frac{\alpha}{s_{2}^{2}}=\alpha \frac{\left(s_{1}+s_{2}\right)\left(s_{1}-s_{2}\right)}{s_{1}^{2} s_{2}^{2}}
$$

we need only consider the expression $\left|s_{1}-s_{2}\right|(|W(1, t)|+|W(0, t)|)$. But the terms in this expression are estimated like the terms in $I_{1}$. Hence, with the exception of the $Z^{2}(1, t)$ term, $\left|I_{3}\right|$ is bounded by the right-hand side of (4.20).

For $I_{4}$ we need to estimate

$$
\left|s_{1}-s_{2}\right| \int_{0}^{1}\left|W_{\xi}(\xi, t) \| U_{2, \xi}(\xi, t)\right| d \xi
$$

Applying the Schwarz inequality and using the assumption that $\int_{0}^{1} U_{2, \xi}^{2} d t$ is uniformly bounded in $t$, we obtain

$$
\left|s_{1}-s_{2}\right| \int_{0}^{1}\left|W_{\xi}(\xi, t)\right|\left|U_{2, \xi}(\xi, t)\right| d \xi \leq C(\varepsilon)\left|s_{1}-s_{2}\right|^{2}+\varepsilon \int_{0}^{1} W_{\xi}^{2}(\xi, t) d \xi .
$$


Applying (4.14), we obtain

$$
\begin{aligned}
\left|I_{4}\right| \leq C(\varepsilon)\left\{\left|b_{1}-b_{2}\right|^{2}+\left|\delta_{1}-\delta_{2}\right|^{2}\right. & +\left\|\nu_{1}-\nu_{2}\right\|_{v^{*}}^{2}+\left\|F_{1}-F_{2}\right\|_{u^{*}}^{2} \\
& \left.+\int_{0}^{t} W^{2}(1, \tau) d \tau+\int_{0}^{t} Z^{2}(1, \tau) d \tau\right\} \\
& +\varepsilon \int_{0}^{1} W_{\xi}^{2}(\xi, t) d \xi,
\end{aligned}
$$

where we have used the Schwarz inequality on (4.14).

By an analysis similar to the estimation of $I_{4}$ we find that

$$
\left|I_{5}\right| \leq C\left\{\left|s_{1}-s_{2}\right|^{2}+\left|\dot{s}_{1}-\dot{s}_{2}\right|^{2}+\int_{0}^{1} W^{2}(\xi, t) d \xi\right\}
$$

and, applying (4.14) and (4.13), we obtain

$$
\begin{aligned}
\left|I_{5}\right| \leq C\{\mid & \left|b_{1}-b_{2}\right|^{2}+\left|\delta_{1}-\delta_{2}\right|^{2}+\left\|\nu_{1}-\nu_{2}\right\|_{v^{*}}^{2} \\
& +\left\|F_{1}-F_{2}\right\|_{u^{*}}^{2}+W^{2}(1, t)+Z^{2}(1, t) \\
& \left.+\int_{0}^{t}\left[W^{2}(1, \tau)+Z^{2}(1, \tau)\right] d \tau+\int_{0}^{1} W^{2}(\xi, t) d \xi\right\} .
\end{aligned}
$$

Combining the estimates for $I_{1}$ through $I_{5}$ we obtain

$$
\begin{aligned}
& \frac{1}{2} \frac{d}{d t} \int_{0}^{1} W^{2}(\xi, t) d \xi+\frac{\alpha}{s_{1}^{2}} \int_{0}^{1} W_{\xi}^{2}(\xi, t) d \xi \\
& \leq C(\varepsilon)\left\{\left|b_{1}-b_{2}\right|^{2}+\left|\delta_{1}-\delta_{2}\right|^{2}+\left\|\nu_{1}-\nu_{2}\right\|_{v^{*}}^{2}+\left\|F_{1}-F_{2}\right\|_{u^{*}}^{2}\right. \\
&+\left|\gamma_{1}-\gamma_{2}\right|^{2}+\left\|f_{1}-f_{2}\right\|_{T}^{2} \\
&+W^{2}(1, t)+W^{2}(0, t)+Z^{2}(1, t)+\int_{0}^{1} W^{2}(\xi, t) d \xi \\
&\left.+\int_{0}^{t} W^{2}(1, \tau) d \tau+\int_{0}^{t} Z^{2}(1, \tau) d \tau\right\}+\varepsilon \int_{0}^{1} W_{\xi}^{2}(\xi, t) d \xi
\end{aligned}
$$


Using the trace inequalities for $W^{2}(1, t), W^{2}(0, t)$, and $Z^{2}(1, t)$ with $\varepsilon=\eta$ and using the trace inequalities on $\int_{0}^{t} W^{2}(1, \tau) d \tau$ and $\int_{0}^{1} Z^{2}(1, \tau) d \tau$ with, say, $\varepsilon=\frac{1}{2}$, we obtain

$$
\begin{aligned}
\frac{1}{2} \frac{d}{d t} \int_{0}^{1} W^{2}(\xi, t) d \xi+\frac{\alpha}{s_{1}^{2}} \int_{0}^{1} W_{\xi}^{2}(\xi, t) d \xi & \left.+\left|\gamma_{1}-\gamma_{2}\right|^{2}+\left\|f_{1}-f_{2}\right\|_{T}^{2}\right\} \\
\leq & C(\varepsilon)\left\{\left|b_{1}-b_{2}\right|^{2}+\left|\delta_{1}-\delta_{2}\right|^{2}+\left\|\nu_{1}-\nu_{2}\right\|_{v^{*}}^{2}+\left\|F_{1}-F_{2}\right\|_{u^{*}}^{2}\right. \\
& +C(\varepsilon) C(\eta)\left[\int_{0}^{1} W^{2}(\xi, t) d \xi+\int_{0}^{1} Z^{2}(\xi, t) d \xi\right] \\
& +(C(\varepsilon) \eta+\varepsilon) \int_{0}^{1} W_{\xi}^{2}(\xi, t) d \xi \\
& +C(\varepsilon) \eta \int_{0}^{1} Z_{\xi}^{2}(\xi, t) d \xi \\
& +C(\varepsilon) \int_{0}^{t} \int_{0}^{1}\left(W^{2}(\xi, \tau)+Z^{2}(\xi, \tau)+W_{\xi}^{2}(\xi, \tau)+Z_{\xi}^{2}(\xi \tau)\right) d \xi d \tau .
\end{aligned}
$$

By a similar analysis applied to the $Z$ equation and combining inequalities we finally obtain

$$
\begin{aligned}
& \frac{1}{2} \frac{d}{d t} \int_{0}^{t}\left[W^{2}(\xi, t)+Z^{2}(\xi, t)\right] d \xi+\int_{0}^{1}\left[\frac{\alpha}{s_{1}^{2}} W_{\xi}^{2}(\xi, t)+\frac{\beta}{s_{1}^{2}} Z_{\xi}^{2}(\xi, t)\right] d \xi \\
& \leq C(\varepsilon)\left\{\left|b_{1}-b_{2}\right|^{2}+\left|\delta_{1}-\delta_{2}\right|^{2}+\left\|\nu_{1}-\nu_{2}\right\|_{v^{*}}^{2}+\left\|F_{1}-F_{2}\right\|_{u^{*}}^{2}\right. \\
& \left.+\left|\gamma_{1}-\gamma_{2}\right|^{2}+\left\|f_{1}-f_{2}\right\|_{T}^{2}+\left\|g_{1}-g_{2}\right\|_{T}^{2}\right\} \\
& +C(\varepsilon) C(\eta)\left\{\int_{0}^{1} W^{2}(\xi, t) d \xi+\int_{0}^{1} Z^{2}(\xi, t) d \xi\right\} \\
& +(C(\varepsilon) \eta+\varepsilon)\left\{\int_{0}^{1} W_{\xi}^{2}(\xi, t) d \xi+\int_{0}^{1} Z_{\xi}^{2}(\xi, t) d \xi\right\} \\
& +C(\varepsilon) \int_{0}^{t} \int_{0}^{1}\left(W^{2}(\xi, \tau)+Z^{2}(\xi, \tau)+W_{\xi}^{2}(\xi, \tau)+Z_{\xi}^{2}(\xi \tau)\right) d \xi d \tau
\end{aligned}
$$

Letting $\lambda=\min \left(\frac{\alpha}{s_{1}^{2}}, \frac{\beta}{s_{1}^{2}}\right)$, we can choose $\varepsilon=\frac{\lambda}{4}$ and $\eta$ so small that $C(\varepsilon) \eta=\frac{\lambda}{4}$ to obtain

$$
\begin{aligned}
& \frac{1}{2} \frac{d}{d t} \int_{0}^{1}\left[W^{2}(\xi, t)+Z^{2}(\xi, t)\right] d \xi+\frac{\lambda}{2} \int_{0}^{1}\left[W_{\xi}^{2}(\xi, t)+Z_{\xi}^{2}(\xi, t)\right] d \xi \\
& \leq C(\varepsilon)\left\{\left|b_{1}-b_{2}\right|^{2}+\left|\delta_{1}-\delta_{2}\right|^{2}+\left\|\nu_{1}-\nu_{2}\right\|_{v^{*}}^{2}+\left\|F_{1}-F_{2}\right\|_{u^{*}}^{2}\right. \\
& \left.\quad+\left|\gamma_{1}-\gamma_{2}\right|^{2}+\left\|f_{1}-f_{2}\right\|_{T}^{2}+\left\|g_{1}-g_{2}\right\|_{T}^{2}\right\} \\
& \quad+C(\varepsilon) C(\eta)\left\{\int_{0}^{1} W^{2}(\xi, t) d \xi+\int_{0}^{1} Z^{2}(\xi, t) d \xi\right\} \\
& \quad+C(\varepsilon) \int_{0}^{t} \int_{0}^{1}\left(W^{2}(\xi, \tau)+Z^{2}(\xi, \tau)+W_{\xi}^{2}(\xi, \tau)+Z_{\xi}^{2}(\xi \tau)\right) d \xi d \tau .
\end{aligned}
$$


Selecting the minimum of $\frac{1}{2}$ and $\frac{\lambda}{4}$, and reducing the left-hand side of (4.28), and dividing, we obtain

$$
y^{\prime}(t) \leq C_{1} \Delta+C_{2} y(t)+C_{3} \int_{0}^{t} y(\tau) d \tau
$$

where

$$
\begin{gathered}
y(t)=\int_{0}^{1}\left[W^{2}(\xi, t)+Z^{2}(\xi, t)\right] d \xi+\int_{0}^{t} \int_{0}^{1}\left[W_{\xi}^{2}(\xi, \tau)+Z_{\xi}^{2}(\xi, \tau)\right] d \xi d \tau \\
\Delta=\left|b_{1}-b_{2}\right|^{2}+\left|\delta_{1}-\delta_{2}\right|^{2}+\left\|\nu_{1}-\nu_{2}\right\|_{v^{*}}^{2}+\left\|F_{1}-F_{2}\right\|_{u^{*}}^{2} \\
+\left|\gamma_{1}-\gamma_{2}\right|^{2}\left\|f_{1}-f_{2}\right\|_{T}^{2}+\left\|g_{1}-g_{2}\right\|_{T}^{2}
\end{gathered}
$$

and where we have increased the right-hand side of (4.28) by the addition of a constant times the term

$$
\int_{0}^{t} \int_{0}^{\eta} \int_{0}^{1}\left[W_{\xi}^{2}(\xi, \tau)+Z_{\xi}^{2}(\xi, \tau)\right] d \xi d \tau d \eta .
$$

Integrating from 0 to $t$ and noting that $y(0)=\left\|\phi_{1}-\phi_{2}\right\|^{2}+\left\|\psi_{1}-\psi_{2}\right\|^{2}$, where $\|\cdot\|$ denotes the $L^{2}$ norm with respect to the spatial variable, and that $y(t) \geq 0$, we see that

$$
\begin{aligned}
0 \leq y(t) & \leq C_{1} \Delta_{1}+C_{4} \int_{0}^{t}(t-\tau+1) y(\tau) d \tau \\
& \leq C_{1} \Delta_{1}+C_{5} \int_{0}^{t} y(\tau) d \tau,
\end{aligned}
$$

where

$$
\Delta_{1}=\Delta+\left\|\phi_{1}-\phi_{2}\right\|^{2}+\left\|\psi_{1}-\psi_{2}\right\|^{2} .
$$

By Gronwall's inequality we find that

$$
y(t) \leq C_{1} \Delta_{1} e^{C_{5} T}
$$

Thus we can state the following result.

THEOREM. Under the assumption that the solutions $(U, V, s)$ to problem $(4.1)-(4.2)$ satisfy $\int_{0}^{1} U_{\xi}^{2}(\xi, t) d \xi \leq C$ and $\int_{0}^{1} V_{\xi}^{2}(\xi, t) d \xi \leq C$ uniformly with respect to $t$, we obtain for two triples $\left(U_{i}, V_{i}, s_{i}\right), i=1,2$, the estimate

$$
\begin{aligned}
& \int_{0}^{1}\left[U_{1}(\xi, t)-U_{2}(\xi, t)\right]^{2}+\left[U_{1}(\xi, t)-U_{2}(\xi, t)\right]^{2} d \xi \\
&+\int_{0}^{1}\left[U_{1, \xi}(\xi, t)-U_{2, \xi}(\xi, t)\right]^{2}+\left[U_{1, \xi}(\xi, t)-U_{2, \xi}(\xi, t)\right]^{2} d \xi \\
& \leq C \Delta_{1},
\end{aligned}
$$

where the constant $C$ depends only on the a priori bounds on the data and the a priori bounds on the solutions obtained in Sec. 2 and where $\Delta_{1}$ is defined by (4.33) and (4.31).

Proof. See the analysis preceding the statement of the theorem. 
We remark that if $f$ and $g$ are continuous in $t$ for $0 \leq t \leq T$ and if $\phi$ and $\psi$ are continuously differentiable in $x$ on $0 \leq x \leq b$, then we obtain a priori bounds on $\left|U_{\xi}\right|$ and $\left|V_{\xi}\right|$ that are uniform in $t$ (see [C]).

Since problem (1.1)-(1.2) is equivalent to problem (4.1)-(4.2), we have obtained unicity and continuous dependence upon the data for problem (1.1)-(1.2), thus completing the proof of the main theorem under the additional assumptions contained in the remark above.

In closing, we note again that the assumptions leading to a proof of existence of solutions to problem (1.1)-(1.2) are weaker than the assumptions that we have used to prove unicity and continuous dependence upon the data. It would be of interest to resolve this discrepency.

Finally, by refining the analysis above, differences in the parameters $\alpha$ and $\beta$ can be incorporated, leading to the conclusion that solutions also depend continuously on these parameters.

\section{REFERENCES}

[C] John. R. Cannon, The one-dimensional heat equation, Encyclopedia of Mathematics, AddisonWesley, Reading, MA, 1984

[CCF] John R. Cannon, James C. Cavendish, and Antonio Fasano, A free boundary-value problem related to the combustion of a solid, SIAM J. Appl. Math. 45 798-809 (1985)

[CL] John R. Cannon and Yanping Lin, A free boundary-value problem related to the combustion of a solid: first initial-boundary value case, Proc. of the Intl. Conf. on Theory and Appl. of Diff. Eq. (Reza Aftabizadeh, ed.), Ohio University Press, Athens, Ohio, 1989, pp. 109-121

[F] A. Fasano, A free boundary value problem in combustion, Nonlinear parabolic equations: qualitative properties of solutions, Pitman Research Notes, Math Series, Longman Sci. Tech., Harlow, 1987

[FR] A. Friedman, Free boundary problem for parabolic equations: I. Melting of solids, J. Math. Mech. 8 499-517 (1959)

[LC] Xhi Yuan Liang and Hong Cheng, $A$ class of free boundary problems with two boundaries arising in combustion of solids, J. Harbin Inst. Tech. 24 1-6 (1992) 